\title{
A Novel Way for Determining Bravais Lattice Using a Single Electron Backscatter Diffraction Pattern
}

\author{
Lili Li, Ming Han \\ School of Materials Science and Engineering, Fujian University of Technology, Fuzhou, 350118, China
}

An electron backscatter diffraction (EBSD) pattern always provides abundant crystallographic information but disappointingly low accuracy. A typical EBSD pattern usually contains dozens of visible Kikuchi bands that intersect each other to form over a hundred Kikuchi poles. The poles correspond to zone axes in real space or lattice planes in reciprocal space. The band widths are inversely proportional to the interplanar spacings of diffracting lattice planes, and the angle formed by the beam source and two band center-lines approximately corresponds to angle between two lattice planes. However, the band width measurement has a relative error of 5-20\% [1] due to the complex profile. In addition, EBSD patterns always suffer from gnomic distortions [2].

Compensating the poor accuracy by the large amount of crystallographic information, a computer program EBSDL has been successfully developed to determine Bravais lattice of unknown crystals using a single EBSD pattern. The pattern center and the detector distance of the pattern as well as the accelerating voltage used are needed to execute EBSDL.

Patterns from omphacite, diopside and ilmenite mineral crystals with non-cubic structure will be demonstrated as test cases to show the determining process and results. After manually detecting the bands from the patterns, EBSDL will automatically complete all calculations and give a solution with Bravais lattice type and parameters. Figure 1 presents the interface of EBSDL with an EBSD pattern that is determined to be omphacite with base-centered monoclinic. Also shown are a result dialog and a wizard dialog that orients users to the next step according to current status during the whole operation process. It should be pointed out that the lattice parameters determined from EBSD patterns appear to disregard the atomic type, suggesting that ordering of structure does not influence the EBSD patterns. Detailed analysis for the accuracy of the determination will be discussed.

The program EBSDL is based on the Kikuchi bands detected from the pattern, independent from any $a$ priori information about its crystal structure. The algorithm is also unrelated to any crystal symmetry information and is therefore applicable to all crystal systems. The application of the novel program is expected to endow scanning electron microscopy with the feasibility to directly determine Bravais lattice of unknown crystals in addition to the conventional imaging of the surface morphology.

\section{References:}

[1] D Dingley and S Wright, J. Appl. Cryst. 42 (2009), p. 234-241.

[2] K Baba-kishi, Scanning 20 (1998), p. 117-127. 




Figure 1. A screen capture showing the graphic interface of the EBSDL with an opened test EBSD pattern that is determined to be omphacite. The dialog in the upper left corner is the wizard that floats on the interface during the whole process and is moveable by using mouse. The result dialog in the lower left corner presents the solution as well as the current, average and maximum errors of the bands and poles. The indices bands and poles that is the nearest from the cursor as well as corresponding error information can be instantly updated as the mouse moves on the pattern. The dialog in the upper right shows an as-measured reciprocal lattice plane and the one in the lower right presents the corresponding corrected counterpart associated with fitting grids. The vector that is closest to the cursor position is shown in red, and the error information and the ordinal number of the plane are shown in the panes of the status bar. 